\title{
Optimalizing Control in the Presence of Noise Interference'
}

\author{
SEDAT SERDENGECTI ${ }^{2}$
}

\author{
Daniel and Florence Guggenheim Jet Propulsion Center, California Institute of Technology, Pasadena, Calif.
}

\begin{abstract}
The performance of a modified peak-holding optimalizing control system depends in part on the ability of the controller to detect the input signal frequency component in the output of the controlled system. This paper describes several techniques that may be used for detecting this component and analyzes one of them in great detail. The subject of the detailed analysis is the method of filtering through cross-correlation. A statistical analysis is also carried out in order to demonstrate the efficiency of this method.
\end{abstract}

\section{Introduction}

N THE peak-holding type of optimalizing controller, which was introduced by C. S. Draper and Y. T. Li (1), ${ }^{3}$ the two basic functions of an optimalizing control system are combined. One is the detection of the input setting that yields the maximum output. The second is the adjustment of the input to make it agree with the optimum setting, thereby actually maximizing the output. For example, the peak-holding optimalizing controller may include an output sensing instrument whose function is to follow the output when the output is increasing but to hold the maximum value of the output when the output is decreasing. The input drive unit of the control system reverses its direction whenever a fixed critical difference exists between the reading of the output sensing instrument and the output itself. In this manner the input is forced to hunt about the optimum input setting. The resultant hunting frequency of the input is a function of the variations in the input-output relationship of the controlled system. The input hunting frequency will only be constant when this relationship is constant, and in such a case the optimalizing control is unnecessary because the system can be easily calibrated once and for all. The dynamic analysis of the peakholding type of system for a constant input-output relationship was considered by $\mathrm{H}$. S. Tsien and the author in a previous paper (2).

In the presence of noise interference, the above method of detecting the maximum of the input-output relationship is not useful. Because of superimposed noise on the "actual" output, the output sensing instrument would never indicate the true optimum state of the controlled system. Furthermore, the input reversal instants would occur earlier or later, in a random manner, depending on whether the magnitude of the output with superimposed noise was greater or less than the magnitude of the "actual" output. It is clear that the noise interference effects would result in a random fluctuation in the input hunting frequency of the system. Thus the true optimum state of the controlled system might never be reached, and the system would fail to accomplish the original purpose of the optimalizing control: to constrain the system near the optimum state.

Since the purpose is to operate near the optimum state in spite of the "drift" of the system and of the noise interference, one may modify the peak-holding type of system by fixing

Received July 24, 1955.

1 This paper is based on part of a thesis submitted by the author for partial fulfillment of the requirements of $\mathrm{Ph}$.D. in Mechanical Engineering, California Institute of Technology.

${ }^{2}$ Research Fellow in Jet Propulsion.

${ }^{3}$ Numbers in parentheses indicate References at end of paper. the input hunting frequency and by specifying the input drive speed. Thus the input drive unit of the modified controller functions completely independent of the input-output relationship of the controlled system and of the noise interference. We now have two distinct problems to solve. First, a procedure must be devised for detecting whether the system is in the optimum state (correct mode of operation) or not. Second, on the basis of the amount of incorrect mode of operation, the system must be driven to the optimum state by the proper input adjustments and kept there with a prescribed minimum error in the presence of noise. Since the input adjustment depends on the outcome of the detection process it is obvious that the longer the latter takes, the slower the former will be. However, there is a limit to how slow the input adjustment can be and still permit the controller to keep up with the constantly shifting input-output relationship of the controlled system. In practice this means that the input hunting frequency in the case of the modified peak-holding controller will nearly always be higher than the nominal hunting frequency of a peakholding controller when both are to be used for the same application.

The detection of the mode of operation of the modified peak-holding type of controller may be based on the following facts. We observe from Fig. 1 (A) that the correct or optimal mode of operation of this controller is achieved when the output hunts symmetrically about the point of maximum output. In this case the time variation of output is independent of whether the input is increasing or decreasing. Consequently, the output will fluctuate with twice the frequency of the input. The correct mode of operation may be characterized therefore by the fact that the output has no Fourier component of frequency equal to that of the input. The other diagrams of Fig.
(B)

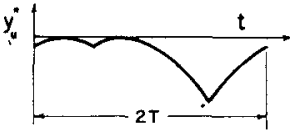

$(|b|<a ; b<0)$

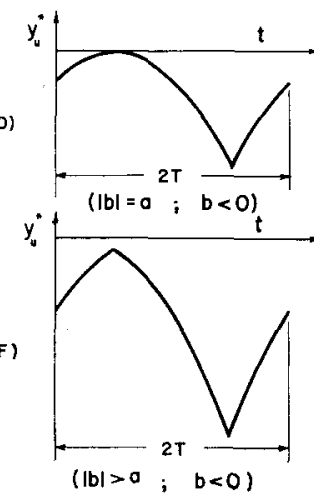

(c)

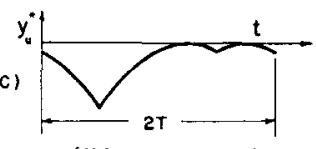

$(|b|<a ; b>0)$

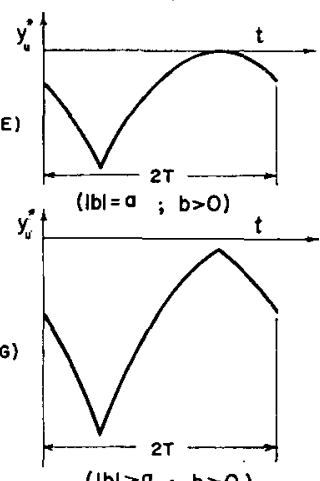

Fig. 1 Performance of an ideal peak-holding optimalizing control system for various amounts of incorrect input 
1 show the output variation when the system is operating in various incorrect modes; it will be noted that the output contains some component of the input frequency for each of these incorrect modes. However, the foregoing facts are based on the existence of a parabolic relationship between the input and the output in the neighborhood of the optimum output. Although it may still be possible to apply the optimalizing principle when a small amount of asymmetry is contained in the input-output characteristic relationship, the hunting of the output will not be symmetrical about the optimum point.

We shall begin our analysis by indicating a few procedures for detecting the presence of the fundamental frequency or input hunting frequency component in the output of the system.

The author is grateful for his opportunity to prepare this work with the guidance of H. S. Tsien, whose continued interest and very kind criticisms were essential for the success of this investigation.

\section{Detection Procedures}

The difference between correct and incorrect modes of operation, as indicated in the introduction, is the occurrence of the first harmonic component in the output signal in the former case and the occurrence of both the fundamental and the first harmonic components in the output signal in the latter case. To illustrate the detection procedures, then, one could assume the noise-free output signal in the incorrect mode of operation to be

$$
y=A+B \sin w_{i} t+C \cos w_{i} t+D \sin 2 w_{i} t+E \cos 2 w_{i} t
$$

where $w_{i}$ is the circular input hunting frequency. In the following sections, it will be seen that the form of the output $y$ given by this equation is exactly that of the output from a sinusoidal input optimalizing controller in the incorrect mode of operation. The coefficients $D$ and $E$ of the first harmonic component are only functions of the dynamic characteristics of the controlled system. The coefficients $B$ and $C$ of the fundamental component are proportional to the amount of incorrect input $b$ and are also functions of the dynamic characteristics of the controlled system. The $\mathrm{d}-\mathrm{c}$ component $A$ is a function of both the incorrect input and the dynamic characteristics of the controlled system.

Since the detection process must be accomplished under conditions of noise interference, the detection scheme must reduce the noise interference effects as much as possible. Such detection procedures fall in one or the other of two distinct types.

\section{(A) Direct Sampling and Averaging Technique}

From observations on Fig. 1, for an ideal optimalizing control with input parameters $a$ and $b$, one can summarize the following facts:

1 When $b<a$, and when $b=0$ (correct operation), two minimum points of the "potential" output occur during each input hunting cycle, and are located at the input reversal instants. In these cases, the maximum points of the "potential" output are located symmetrically with respect to the minimum points.

2 When $b \geq a$, there is always one minimum and one maximum point of the "potential" output per input cycle, and these are located at the input reversal instants.

When the input and output linear group characteristic time constants, $\tau_{i}$ and $\tau_{0}$, respectively, of the controlled system are small compared to the output hunting period $T$ of the system in the correct mode of operation, the conclusions mentioned above remain unchanged, with the exception of the locations of the maximum and minimum points. However, one must give special care to the first case. For instance, when $b<a$, there occurs only one minimum and one maximum point per input cycle when the characteristic time constants $\tau_{i}$ and $\tau_{0}$ are of the same order of magnitude as $T$, or larger. Thus for small values of $\tau_{i}$ and $\tau_{0}$, one can conclude that the locations of the minimum points of the output are independent of the amount of incorrect input $b$, and depend only on the dynamic characteristics of the controlled system. On the other hand, the locations of the maximum points are dependent on the amount of incorrect input $b$ when $b<a$, and are independent of the amount of incorrect input $b$ when $b \geq a$. Therefore the method in this case would be to locate a minimum point of the output signal and to investigate whether or not a maximum point of the output occurs after $T / 2$ sec. When the maximum point of the output occurs $T / 2$ sec after the occurrence of the minimum point, the control system is in the correct mode of operation. Otherwise the input level must be changed to produce the desired condition.

To locate either a minimum or a maximum of the output signal under noise interference, consider two sets of $N$ samples. One set is taken at the time instants

$$
t=0,2 T, 4 T, \ldots, 2(N-1) T
$$

and the other set is taken at the instants

$$
t=\tau, \tau+2 T, \tau+4 T, \ldots, \tau+2(N-1) T
$$

where $\tau \leq T / 2$. One can choose $N$ such that when average values of these samples are considered, the noise interference effects are made sufficiently small. Denoting the average of the first set of samples by $\bar{y}_{1}$ and that of the second by $\bar{y}_{2}$, one can distinguish the following three cases:

(a) When $\bar{y}_{1}<\bar{y}_{2}$, the maximum point is behind and the minimum point is ahead of the sampling instants.

(b) When $\bar{y}_{1}>\bar{y}_{2}$, the maximum point is ahead and the minimum point is behind the sampling instants.

(c) When $\bar{y}_{1}=\bar{y}_{2}$, there occurs either a maximum or a minimum point of the output which is located approximately half way between two sampling instants.

By displacing the sampling instants, one can always obtain case (c). The choice of $\tau$ must be such that two sets of samples are distinguishable when samples are taken as in the cases (a) and (b), and such that when case (c) occurs the maximum or the minimum point is located very nearly half way between two sampling instants.

\section{(B) Explicit Detection of the Fundamental Component}

The following three methods may be considered as representative examples of this class:

1 Narrow Band-Pass Filters. Any convenient filter in this category can be utilized. The detection criterion in this case is to tune the filter circuit on the fundamental component frequency and to minimize the measured amplitude of the fundamental component by varying the input level.

2 Direct Integration Technique. Starting at any time origin $t=0$, the output signal is integrated with respect to time for a complete cycle of the first harmonic component. The next integration is performed $\Delta T$ sec after the previous integration has been terminated, and so on. Thus the integrations are performed starting at time instants

$$
t=0, T+\Delta T, 2(T+\Delta T), \ldots,(N-1)(T+\Delta T)
$$

and complete data have been obtained when $N \Delta T=2 T$.

When the starting instants of the integration procedure are displaced, the resulting integrals have approximately the following form

$I_{N}=C \sin \left[w_{i} N(\Delta T)+\Phi\right] \quad[N=0,1,2, \ldots,(2 T / \Delta T)-1]$

where $C$ and $\Phi$ are constants. The noise interference effects are somewhat suppressed by the integration process and there is no contribution to the integrals from higher harmonics. When $N$ equivalent integrators are used for this process, a complete set of data is available in $2 T$ sec, since each integration process can start $\Delta T$ sec after the preceding one ends.

The criterion in this case is that the amplitude $C$ of $I_{N}$ should be a minimum which is reached by varying the input 
level. In this condition the system is as close to the correct mode of operation as possible.

3 Filtering by Correlation Method. This method uses the principle of correlating the output signal with a sinusoidal signal of fundamental frequency $w_{i}$. The details of this procedure will be given in the following sections.

The preceding discussions indicate only a few procedures of many possible ways of detecting and eliminating the incorrect modes of operation of the modified optimalizing control system. The primary objective in the following sections is to demonstrate a statistical analysis designed to assess the merit of the proposed method of filtering by correlation. The ultimate choice of the design has to be based upon such an analysis for each of the various possible methods and upon other engineering factors.

\section{Mathematical Analysis of the Incorrect Mode of Operation}

To determine the dynamic effects of the controlled system on the performance of the modified optimalizing control system, neglecting noise interference effects, a modified optimalizing controller with an incorrect input will be analyzed.

In a similar manner as in the previous paper by Tsien and the author (2), the "potential" input $x_{u}{ }^{*}$, specified as a sawtooth curve with period $2 T$, amplitude $a$, and an amount of incorrect input $b$ can be expanded into a Fourier series as

$x_{u}{ }^{*}=b+\frac{8 a}{\pi^{2}} \sum_{n=0}^{\infty} \frac{(-1)^{n}}{(2 n+1)^{2}} \frac{1}{2 i}\left[e^{\frac{2 n+1}{2} i w_{0} t}-e^{\frac{2 n+1}{2} i w_{0} t}\right]$.

where $w_{0}$ is the circular output hunting frequency in the correct mode of operation and is defined as

$$
w_{0}=2 \pi / T=2 w_{i}
$$

In operator form, the actual input $x_{u}$ and the actual output $y_{u}$ are obtained as

$$
\begin{array}{r}
x_{u}=b+\frac{8 a}{\pi^{2}} \sum_{n=0}^{\infty} \frac{(-1)^{n}}{(2 n+1)^{2}} \frac{1}{2 i}\left[F_{i}\left(\frac{2 n+1}{2} i w_{0}\right) e^{\frac{2 n+1}{2} i w_{0} t}-\right. \\
\left.F_{i}\left(-\frac{2 n+1}{2} i w_{0}\right) e^{-\frac{2 n+1}{2} i w_{0} t}\right] \ldots
\end{array}
$$

and

$$
\begin{gathered}
y_{u}=-k b^{2}+\frac{8 a b k}{\pi^{2}} i \sum_{n=0}^{\infty} \frac{(-1)^{n}}{(2 n+1)^{2}}\left[F_{0}\left(\frac{2 n+1}{2} i w_{0}\right) \times\right. \\
F_{i}\left(\frac{2 n+1}{2} i w_{0}\right) e^{\frac{2 n+1}{2} i w_{0} t}-F_{0}\left(-\frac{2 n+1}{2} i w_{0}\right) \times \\
\left.F_{i}\left(-\frac{2 n+1}{2} i w_{0}\right) e^{-\frac{2 n+1}{2} i w_{0} t}\right]+\frac{16}{\pi^{4}} a^{2} k \sum_{n=0}^{\infty} \sum_{m=0}^{\infty} \times \\
\frac{(-1)^{n+m}}{(2 n+1)^{2}(2 m+1)^{2}}\left[F_{0}\left\{(n+m+1) i w_{0}\right\} F_{i}\left(\frac{2 n+1}{2} i w_{0}\right) \times\right. \\
F_{i}\left(\frac{2 m+1}{2} i w_{0}\right) e^{(n+m+1) i w_{0} t}-F_{0}\left\{(n-m) i w_{0}\right\} \times \\
F_{i}\left(\frac{2 n+1}{2} i w_{0}\right) F_{i}\left(-\frac{2 m+1}{2} i w_{0}\right) e^{(n-m) i w_{0} t}- \\
F_{0}\left\{-(n-m) i w_{0}\right\} F_{i}\left(-\frac{2 n+1}{2} i w_{0}\right) F_{i}\left(\frac{2 m+1}{2} i w_{0}\right) \times \\
e^{-(n-m) i w_{0} t}+F_{0}\left\{-(n+m+1) i w_{0}\right\} F_{i}\left(-\frac{2 n+1}{2} i w_{0}\right) \times \\
\left.F_{i}\left(-\frac{2 m+1}{2} i w_{0}\right) e^{-(m+n+1) i w_{0} t}\right] \ldots \ldots
\end{gathered}
$$

Comparison of Equations [2] and [3] indicates that the output $y_{u}$ has the same hunting period as the input $x_{u}$. The hunting loss $D_{u}$ of this incorrectly operating control system is the time average of the output $y_{u}$; i.e.

$$
\begin{array}{r}
D_{u}=k b^{2}+\frac{32 a^{2} k}{\pi^{4}} \sum_{n=0}^{\infty} \frac{1}{(2 n+1)^{4}} F_{i}\left(\frac{2 n+1}{2} i w_{0}\right) \times \\
F_{i}\left(-\frac{2 n+1}{2} i w_{0}\right) .
\end{array}
$$

It is clear from Equation [4] that the hunting loss $D_{u}$ depends on the magnitude of the incorrect input $b$.

With the assumption that the transfer functions of the input and output linear groups can be represented closely by those for first order systems, i.e.

$$
F_{i}(i w)=1 /\left(1+i w \tau_{i}\right) \ldots
$$

and

$$
F_{0}(i w)=1 /\left(1+i w \tau_{0}\right)
$$

and with the introduction of a dimensionless parameter $l$ for incorrect operation defined as

$$
l=b / a .
$$

the actual output $y_{u}$ and the hunting loss $D_{u}$, given by Equations [3] and [4], respectively, become

$$
\begin{aligned}
& y_{u}=N^{2} T^{2} k\left\{-\left(l^{2} / 4\right)-(-1)^{j} l\left[\left(\frac{t}{T}-j\right)-\left(\frac{\tau_{0}}{T}+\frac{\tau_{i}}{T}\right)+\right.\right. \\
& \left.\left.\frac{\left(\tau_{0} / T\right)^{2} e^{-\left(\frac{t}{T}-j\right) /\left(\tau_{0} / T\right)}}{\left(\frac{\tau_{0}}{T}-\frac{\tau_{i}}{T}\right) \cosh \left(T / 2 \tau_{0}\right)}-\frac{\left(\tau_{i} / T\right)^{2} e^{-\left(\frac{t}{T}-j\right) /\left(\tau_{i} / T\right)}}{\left(\frac{\tau_{\theta}}{T}-\frac{\tau_{i}}{T}\right) \cosh \left(T / 2 \tau_{i}\right)}\right]\right\}+ \\
& 2 N^{2} T^{2} k\left\{-\left[\frac{1}{2}\left(\frac{t}{T}-j\right)^{2}-\left(\frac{\tau_{0}}{T}+\frac{\tau_{i}}{T}\right)\left(\frac{t}{T}-j\right)+\frac{1}{2}\left(\frac{\tau_{i}}{T}\right)^{2}+\right.\right. \\
& \left.\frac{\tau_{i} \tau_{0}}{T^{2}}+\left(\frac{\tau_{0}}{T}\right)^{2}\right]-\frac{1}{2} \frac{\left(\tau_{0} / T\right)^{2}}{\left(\frac{\tau_{0}}{T}-\frac{\tau_{i}}{T}\right)}\left[\frac{2\left(\tau_{i} / T\right)^{3} \tanh \left(T / 2 \tau_{i}\right)}{\left(\frac{\tau_{0}}{T}-\frac{\tau_{i}}{T}\right)\left(2 \frac{\tau_{0}}{T}-\frac{\tau_{i}}{T}\right)}-1\right] \times \\
& \frac{e^{-\left(\frac{t}{T}-j\right) /\left(\tau_{0} / T\right)}}{\sinh \left(T / 2 \tau_{0}\right)}+\left[\left(\frac{t}{T}-j\right)+\frac{\left(\tau_{i} / T\right)^{2}}{\left(\frac{\tau_{0}}{T}-\frac{\tau_{i}}{T}\right)}\right] \times \\
& \frac{\left(\tau_{i} / T\right)^{2} e^{-\left(\frac{t}{T}-j\right) /\left(\tau_{i} / T\right)}}{\left(\frac{\tau_{0}}{T}-\frac{\tau_{i}}{T}\right) \cosh \left(T / 2 \tau_{i}\right)}+\frac{1}{2} \frac{\left(\tau_{i} / T\right)^{3}}{\left(2 \frac{\tau_{0}}{T}-\frac{\tau_{i}}{T}\right)} \times \\
& \left.\frac{e^{-2\left(\frac{t}{T}-j\right) /\left(\tau_{i} / T\right)}}{\cosh ^{2}\left(T / 2 \tau_{i}\right)}\right\}
\end{aligned}
$$

when

$$
-1 / 2 \leq[(t / T)-j] \leq 1 / 2
$$

where

$$
j=0,1,2, \ldots
$$

and

$$
D_{u}=N^{2} T^{2} k\left[\frac{1}{4}\left(l^{2}+\frac{1}{3}\right)-\left(\tau_{i} / T\right)^{2}+2\left(\tau_{i} / T\right)^{3} \times\right.
$$

where $N=2 a / T$ is again the input drive speed.

Figs. 2 and 3 show the dimensionless plots of the actual output for several particular values of $l$, for the cases when $\left(\tau_{0} / T\right)=$ 0.15 and $\left(\tau_{i} / T\right)=0.10$. The plots in these figures have 


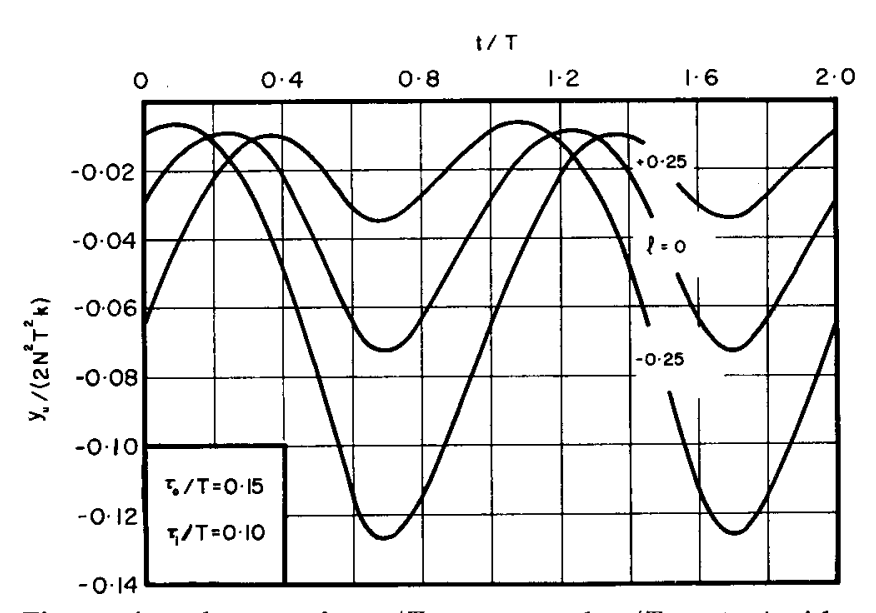

Fig. 2 Actual output for $\tau_{i} / T=0.10$ and $\tau_{0} / T=0.15$ with parameter

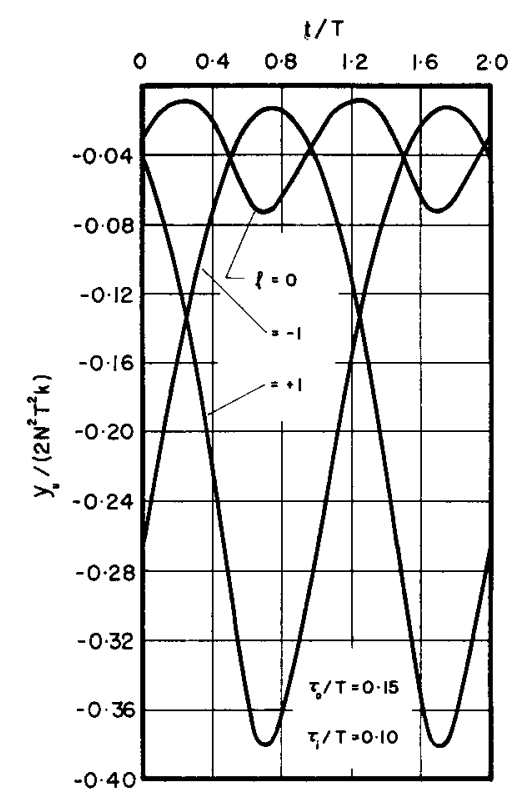

Fig. 3 Actual output for $\tau_{i} / T=0.10$

and $\tau_{0} / T=0.15$ with $l$ parameter

essentially the same shape as those for the corresponding cases illustrated in Fig. 1. The differences are again the rounding of the sharp corners and the time lag due to dynamic effects.

\section{Detection and Elimination of the Incorrect Mode of Operation Without Noise Interference}

The parameter $l$, defined by Equation [7], is introduced in such a manner that it is not only possible to detect the incorrect mode of operation, but it is also possible to determine the magnitude and the sign of the incorrect input $b=(N T / 2) l$. Therefore the problem is to devise a scheme to determine the parameter $l$. As was explained in a previous section, one of the possible schemes is as follows: the actual output $y_{u}$ is cross-correlated with a sinusoid which has an amplitude $A$, frequency $w_{i}$, and a time displacement $\tau$ with respect to the actual output signal $y_{u}$.

In general, the cross-correlation of a function $f(t)$ with $g(t)$ is defined as

$$
\chi(\tau)=\lim _{\theta \rightarrow \infty} \frac{1}{2 \theta} \int_{-\theta}^{\theta} f(t+\tau) \overline{g(t)} d t .
$$

where $\overline{g(t)}$ is the complex conjugate of $g(t)$. For periodic real functions $f(t)$ and $g(t)$, the cross-correlation function given by Equation $[10]$ reduces to

$$
\chi(\tau)=\frac{1}{2 \theta} \int_{-\theta}^{\theta} f(t+\tau) g(t) d t .
$$

where $2 \theta$ is the smallest common period of the two functions $f(t)$ and $g(t)$.

Choose

$$
f(t+\tau)=A \sin w_{i}(t+\tau)
$$

and

$$
g(t)=y_{u}(t)
$$

then the cross-correlation function $\chi(\tau)$ given by Equation [11] becomes

$$
\begin{aligned}
\chi(\tau)=\frac{A}{2} \cos w_{i} \tau & \int_{0}^{2} y_{u}\left(t^{\prime}\right) \sin \pi t^{\prime} d t^{\prime}+ \\
& \frac{A}{2} \sin w_{i} \tau \int_{0}^{2} y_{u}\left(t^{\prime}\right) \cos \pi t^{\prime} d t^{\prime}
\end{aligned}
$$

where the integration variable has been changed to $t^{\prime}=t / T$.

By substitution of Equation [8] into Equation [12] and by performing the integrations, $\chi(\tau)$ is obtained as

$$
\begin{aligned}
\chi(\tau)=\frac{2 A N^{2} k l}{w_{i}^{2}\left[1+\left(w_{i} \tau_{0}\right)^{2}\right]\left[1+\left(w_{i} \tau_{i}\right)^{2}\right]} \times \\
\left\{\left(w_{i}{ }^{2} \tau_{0} \tau_{i}-1\right) \cos w_{i} \tau+w_{i}\left(\tau_{0}+\tau_{i}\right) \sin w_{i} \tau\right\}
\end{aligned}
$$

Thus, with a specified set of characteristics of the controlled and control systems, the amplitude $A$ of the sinusoid, and the value of the cross-correlation function $\chi(\tau)$ for a given value of $\tau$, the parameter $l$ can be computed by using Equation [13]. Fig. 4 shows dimensionless plots of the cross-correlation function for $l=+1$ for various values of $\tau_{i} / T$ and $\tau_{0} / T$.

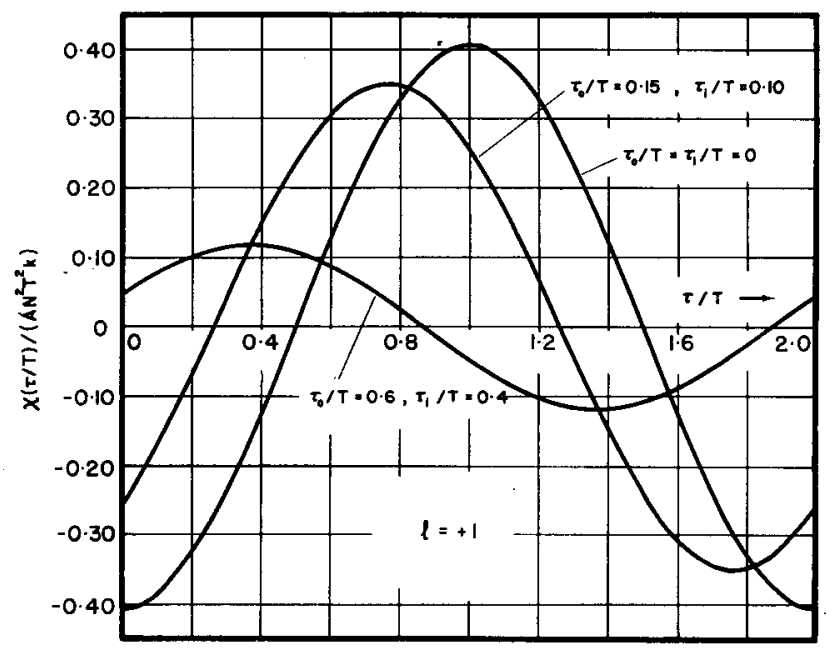

Fig. 4 Dimensionless cross-correlation function $\chi(\tau) /\left(A T^{2} N^{2} k\right)$ vs. $\tau / T$ for various values of $\tau_{i} / T$ and $\tau_{0} / T$ with $l=+1$

It is clear from Equation [13] that there are certain values of time displacement $\tau$ which make the cross-correlation functions vanish regardless of the values of the parameter $l$. Since these values of $\tau$ will not give any information about the incorrect mode of operation, they cannot be utilized. The forbidden values of $\tau$ are found by setting Equation [13] equal to zero, thus

$\left(w_{i} \tau \mp n \pi\right)=\arctan \frac{\left(1-w_{i}{ }^{2} \tau_{0} \tau_{i}\right)}{w_{i}\left(\tau_{0}+\tau_{i}\right)} \quad(n=0,1,2, \ldots)$.

When it is necessary to use a computer for the determination of the parameter $l$, one must choose $\tau$ such that the absolute value of the cross-correlation function is the largest. By differentiating $\chi(\tau)$ with respect to $\tau$ and setting it equal to zero, one obtains the desired values of $\tau$ as

$\left(w_{i} \mp n_{\pi}\right)=\arctan \frac{w_{i}\left(\tau_{0}+\tau_{i}\right)}{\left(w_{i}^{2} \tau_{0} \tau_{i}-1\right)} \quad(n=0,1,2, \ldots)$

When the only information available is the input hunting 
frequency $w_{i}$, Equation [13] can be written as

$$
\chi(\tau)=\alpha \cos w_{i} \tau+\beta \sin w_{i} \tau
$$

where $\alpha$ and $\beta$ are defined as

$$
\begin{aligned}
& \alpha=\frac{2 A N^{2} k l}{w_{i}{ }^{2}} \frac{\left(w_{i}{ }^{2} \tau_{0} \tau_{i}-1\right)}{\left[1+\left(w_{i} \tau_{0}\right)^{2}\right]\left[1+\left(w_{i} \tau_{i}\right)^{2}\right]} \\
& \beta=\frac{2 A N^{2} k l}{w_{i}{ }^{2}} \frac{w_{i}\left(\tau_{0}+\tau_{i}\right)}{\left[1+\left(w_{i} \tau_{0}\right)^{2}\right]\left[1+\left(w_{i} \tau_{i}\right)^{2}\right]}
\end{aligned}
$$

From Equations [17] and [18] it is seen that the coefficient $\beta$ will always have the same sign as the parameter $l$, and the coefficient $\alpha$ will have the same sign as $l$ when $w_{i}{ }^{2} \tau_{0} \tau_{i}>1$, but the opposite sign to $l$ when $w_{i}{ }^{2} \tau_{0} \tau_{i}<1$. When only two distinct values of the cross-correlation function $\chi(\tau)$ are known for two given values of time displacement, except when $\tau_{1}-$ $\tau_{2}=n \pi / w_{i}$, it is possible to determine coefficients $\alpha$ and $\beta$ from Equation [16]. Although there is not enough information to determine the value of the parameter $l$ with either Equation [17] or Equation [18], one knows that the sign of $l$ is the same as that of the coefficient $\beta$. Since the characteristic time constants $\tau_{i}$ and $\tau_{0}$ of the controlled system are not available, the information obtained through the coefficient $\alpha$ is unreliable. Thus knowing only $w_{i}$, the input hunting frequency, and evaluating two distinct values of the cross-correlation function for two distinct values of time displacement, it is still possible to detect the incorrect mode of operation and the direction in which it occurs. To eliminate the incorrect mode of operation in this case, the input level can be varied by increments in accordance with the sign of the coefficient $\beta$. After each stepwise variation of input, two new cross-correlation processes must be carried out. This elimination of the incorrect mode of operation through a trial-and-error technique is continued until both cross-correlations obtained vanish; that is, $l=0$ or $\alpha=\beta=0$. This mode of operation is then the correct mode of operation.

\section{Detection and Elimination of an Incorrect Mode of Operation with Noise Interference}

In the previous section, the detection and elimination criterion of the incorrect mode of operation was formulated for a modified optimalizing control system, free from noise interference effects. Needless to say, noise from both external and internal sources is present and affects the performance of the control system. However, when the noise interference effects are considered, the fundamental concept of the detection and elimination criterion introduced in the previous section is still valid. The method is again to determine a parameter $l^{\prime}$ which is an indication of the amount of the incorrect mode of operation.

For simplicity of analysis, the noise function $n(t)$ is assumed to be superimposed on the actual output $y_{u}(t)$; thus the signal $F(t)$ indicated by an output measuring instrument is of the form

$$
F(t)=y_{u}(t)+n(t)
$$

Then the cross-correlation function $R(\tau)$ of $F(t)$ with a sinusoid of the form $A \sin w_{i}(t+\tau)$ is

$$
R(\tau)=\lim _{\theta \rightarrow \infty} \frac{A}{2 \theta} \int_{-} F(t) \sin w_{i}(t+\tau) d t
$$

By using Equations [12] and [19], an approximate crosscorrelation $R_{m}(\tau)$ depending on a parameter $m$, the number of input cycles over which the correlation process is carried out, can be defined as

$$
R_{m}(\tau)=\chi(\tau)+\frac{A}{2 m T} \int_{0}^{2 m T} n(t) \sin w_{i}(t+\tau) d t \ldots
$$

When a value of the cross-correlation function $R_{m}(\tau)$ and complete control and controlled systems characteristics are available, the parameter $l^{\prime}$, an indication of the incorrect operation mode, can be computed approximately by replacing $\chi(\tau)$ with $R_{m}(\tau)$ in Equation [13]. Thus

$$
l^{\prime}=\frac{w_{i}{ }^{2} R_{m}(\tau)\left[1+\left(w_{i} \tau_{0}\right)^{2}\right]\left[1+\left(w_{i} \tau_{i}\right)^{2}\right]}{2 A N^{2} k\left\{\left(w_{i}{ }^{2} \tau_{0} \tau_{i}-1\right) \cos w_{i} \tau+w_{i}\left(\tau_{0}+\tau_{i}\right) \sin w_{i} \tau\right\}}
$$

The amount of incorrect input which must be eliminated is then $b^{\prime}=(N T / 2) l^{\prime}$. Since the cross-correlation process is carried out for a finite value of $m$, the noise inteference effects are not completely eliminated, and $b^{\prime}$ is not necessarily the actual amount of incorrect input. For example, $l^{\prime}=0$ may not mean that the system is operating in the correct mode. Thus, there is a certain probability of error associated with the value of the parameter $l^{\prime}$.

When the cross-correlation process is carried out for two distinct values of time displacement and only the input hunting frequency $w_{i}$ is known, it is still possible to detect and to eliminate the incorrect operation mode with a certain probability of error due to noise interference effects. In this case the cross-correlation function $R_{m}(\tau)$ can be written as

$$
R_{m}(\tau)=\alpha^{\prime} \cos w_{i} \tau+\beta^{\prime} \sin w_{i} \tau \ldots
$$

With two distinct values of $R_{m}(\tau)$, one can determine the coefficients $\alpha^{\prime}$ and $\beta^{\prime}$ from Equation [22]. Again, with a certain probability of error, one can say that the sign of the parameter $l$ is the same as that of the coefficient $\beta^{\prime}$, and one can vary the input level in increments in order to eliminate the incorrect mode of operation. In this case the information obtained by using the coefficient $\alpha^{\prime}$ is again unreliable.

To refine the process of elimination of the incorrect mode of operation the cross-correlation function $R_{m}(\tau)$, given by Equation [20], must be as free as possible from noise interference effects. One way to reduce noise interference effects is to choose $m$ to be large; that is, to carry out the cross-correlation process for a large number of cycles, and thus reduce the noise interference effects to a minimum by an averaging process. However, the larger the value of $m$ which is chosen, the longer it will take to obtain the desired information.

Another possible way to reduce the noise interference effects is to choose a reasonably small value of $m$ and to carry out the cross-correlation process for several values of time displacement. With these several values of cross-correlation functions, it is possible to produce a cross-correlation function of the form

$$
R_{m}^{*}(\tau)=\alpha^{*} \cos w_{i} \tau+\beta^{*} \sin w_{i} \tau \ldots \ldots \ldots[23]
$$

such that it is the best estimation of Equation [16]. When only one cross-correlator is used, this process is time-consuming, but when it is feasible to use a number of equivalent crosscorrelators, this process takes a rather short time.

One of perhaps several ways of producing Equation [23] through a cross-correlation process for several values of time displacement can be outlined as follows:

Consider a set of values of cross-correlation functions

$$
R_{m_{0}}, R_{m_{1}}, R_{m_{2}}, \ldots, R_{m_{j}}, \ldots, R_{m_{k}}
$$

which correspond to time displacements

$$
0=\tau_{0}, \tau_{1}, \tau_{2}, \ldots, \tau_{j}, \ldots, \tau_{k}=2 \pi / w_{i}
$$

where each of these time displacements differs by an equal increment $\Delta \tau$ from the preceding one. For the value of time displacement $\tau_{j}$, the corresponding error of estimation in cross-correlation function is given by

$$
R_{m_{j}}-R_{m}^{*}\left(\tau_{j}\right)
$$

Since the problem is to fit a simple curve to given data, the method of least squares is considered to be satisfactory. The method of least squares requires that the sum of squares of 
the errors be a minimum. The sum of the squares of the errors $G$ is given by

$$
G=\sum_{j=1}^{k}\left[R_{m_{j}}-\left(\alpha^{*} \cos w_{i} \tau_{j}+\beta^{*} \sin w_{i} \tau_{j}\right)\right]^{2} \ldots[24]
$$

Inspection of Equation [24] shows that the sum of the squares of the errors $G$ is a function of $\alpha^{*}$ and $\beta^{*}$; thus the coefficients $\alpha^{*}$ and $\beta^{*}$ must be determined such that $G$ is a minimum. The requirement for the minimum will be satisfied when the partial derivatives of $G$ with respect to both $\alpha^{*}$ and $\beta^{*}$ vanish, thus the particular values of these coefficients are found to be

$$
\left.\begin{array}{l}
\alpha^{*}=\frac{2}{k} \sum_{j=1}^{k} R_{m_{j}} \cos \frac{2 \pi}{k} j \\
\beta^{*}=\frac{2}{k} \sum_{j=1}^{k} R_{m_{j}} \sin \frac{2 \pi}{k} j
\end{array}\right\}
$$

As was done for the case shown by Equation [21], a parameter $l^{*}$ may be computed to be

$$
l^{*}=\frac{w_{i}{ }^{2} R_{m}{ }^{*}(\tau)\left[1+\left(w_{i} \tau_{0}\right)^{2}\right]\left[1+\left(w_{i} \tau_{i}\right)^{2}\right]}{2 A N^{2} k\left\{\left(w_{i}{ }^{2} \tau_{0} \tau_{i}-1\right) \cos w_{i} \tau+w_{i}\left(\tau_{0}+\tau_{i}\right) \sin w_{i} \tau\right\}}
$$

Fig. 5 shows a complete block diagram of a modified optimalizing control system using a cross-correlator, a variable time displacement sine wave generator, and a computer to filter out the noise interference effects. This control system can be operated to produce either the parameter $l^{\prime}$ by using only one value of time displacement or the parameter $l^{*}$ by using several values of time displacement.

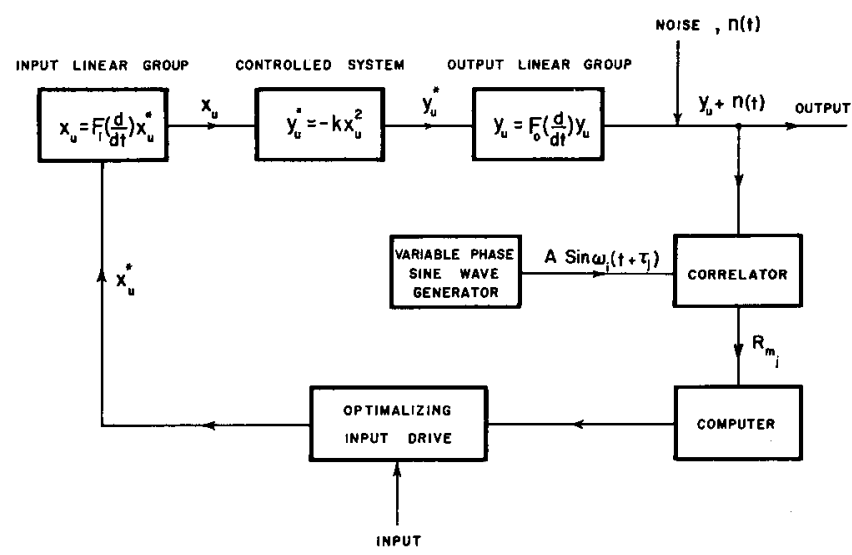

Fig. 5 Block diagram of a modified optimalizing control system with filtering by means of cross-correlation

\section{Probable Error in Detection and Elimination of the Incorrect Mode of Operation in the Pres- ence of Noise Interference}

In the previous section it was shown that with noise interference effects, the criterion for detection and elimination of the incorrect mode of operation can be based either on the parameter $l^{\prime}$ (or $l^{*}$ ) or on the coefficient $\beta^{\prime}$ (or $\left.\beta^{*}\right)^{4}$ depending on how much information is available about the system. Since the parameter $l^{\prime}$ or the coefficient $\beta^{\prime}$ is computed in a finite length of time, the noise interference effects are not completely eliminated, and it is conceivable that the parameter $l^{\prime}$ or the coefficient $\beta^{\prime}$ will contain the following two types of error:

Type I: Error in the magnitude of the parameter $l^{\prime}$ or $\beta^{\prime}$. For example, $l^{\prime}$ and $l$ have the same algebraic signs but their magnitudes differ.

Type II: Error in both algebraic sign and magnitude

${ }^{4}$ In this section the analysis will be carried out for primed quantities. However, the results can be used equivalently for starred quantities. of the parameter $l^{\prime}$ or $\beta^{\prime}$. For example, to obtain $l^{\prime}$ as a negative quantity while $l$ really is a positive quantity, or vice versa.

Type I error is not a serious type of error since the input level is corrected in the right direction. When $l^{\prime}$ is large in magnitude, Type I error is expected to occur more frequently than Type II error. When the magnitude of $l^{\prime}$ is reduced in order to reach the correct mode of operation, both Type I and Type II errors are expected to occur frequently. Type II error is more serious than Type I error, since the occurrence of Type II error is a false alarm, and the input level is changed in the wrong direction. Therefore confining the system as closely as possible to the correct mode of operation results in the frequent occurrence of Type II error. Thus the control system designer must decide upon a certain probability of occurrence of Type II error as being satisfactory, and compute a limiting value of the parameter $l^{\prime}$ or $\beta^{\prime}$ which leads to the best mode of operation. For example, denoting this limiting value of $l^{\prime}$ by $l^{\prime *}$, the computer then should be equipped with a device such that whenever $\left|l^{\prime}\right| \leq\left|l^{\prime *}\right|$, the input level is left unchanged; otherwise alteration of input level is carried on.

In the following analysis, the principal objective is to determine the probability of occurrence of Type II error in the detection and elimination criterion introduced in the previous section. Then a theoretical illustration will be presented so as to demonstrate how the limiting parameters $l^{\prime *}$ and $\beta^{\prime *}$ can be computed.

The probability of occurrence of Type II error is equivalent to the probability of having $l^{\prime}<0$ or $\beta \beta^{\prime}<0$. From Equations [13] and [21] it is clear that the probability of having $l l^{\prime}<0$ is equivalent to the probability of having $x(\tau) R_{m}(\tau)<0$. To compute the probability that $\beta \beta^{\prime}<0$ or $\chi(\tau) R_{m}(\tau)<0$, consider the following relations

$$
\begin{array}{r}
\zeta=\beta \beta^{\prime}=\beta^{\prime 2}-\left(\beta^{\prime}-\beta\right) \beta^{\prime} \ldots \ldots \ldots \ldots \\
\xi=\chi(\tau) R_{m}(\tau)=\left[R_{m}(\tau)\right]^{2}-\left[R_{m}(\tau)-\chi(\tau)\right] R_{m}(\tau) \ldots
\end{array}
$$

from Equations [16], [20], and [22] one can show that

$$
\left.\begin{array}{r}
\beta^{\prime}=\frac{1}{T} \int_{0}^{2 T} R_{m}(\tau) \sin w_{i} \tau d \tau \\
\left(\beta^{\prime}-\beta\right)=\frac{A}{2 T m} \int_{0}^{2 T m} n(t) \cos w_{i} t d t
\end{array}\right\}
$$

By substitution of the expressions given by Equations [20] and [29] into Equations [27] and [28], the relations for the variables $\zeta$ and $\xi$ are found to be

$$
\begin{array}{r}
\zeta=\left[\frac{1}{T} \int_{0}^{2 T} R_{m}(\tau) \sin w_{i} \tau d \tau\right]^{2}-\left[\frac{1}{T} \int_{0}^{2 T} R_{m}(\tau) \sin w_{i} \tau d \tau\right] \times \\
\cdot\left[\frac{A}{2 T m} \int_{0}^{2 T m} n(t) \cos w_{i} t d t\right] \\
\xi=\left[R_{m}(\tau)\right]^{2}-R_{m}(\tau)\left[\frac{A}{2 T m} \int_{0}^{2 T_{m}} n(t) \sin w_{i}(t+\tau) d t\right]
\end{array}
$$

With the assumption that the noise considered in this analysis is a stationary random function and its time average $\overline{n(t)}$ vanishes, the mean values and mean deviations of the statistical variables $\zeta$ and $\xi$ are, respectively

$$
\begin{gathered}
\bar{\zeta}={\beta^{\prime}}^{2}=\left[\frac{1}{T} \int_{0}^{2 T} R_{m}(\tau) \sin w_{i} \tau d \tau\right]^{2} . \\
\bar{\xi}=\left[R_{m}(\tau)\right]^{2} \ldots \ldots \ldots \ldots \ldots
\end{gathered}
$$

and

$$
\begin{aligned}
& \sigma_{\zeta}=\left[\frac{1}{T} \int_{0}^{2 T} R_{m}(\tau) \sin w_{i} \tau d \tau\right] \times \\
& \left\{\frac{A^{2}}{(2 T m)^{2}} \int_{0}^{2 T m} d t^{\prime \prime} \int_{0}^{2 T m} d t^{\prime} \overline{n\left(t^{\prime}\right) n\left(t^{\prime \prime}\right)} \cos w_{i} t^{\prime} \cos w_{i} t^{\prime \prime}\right\}^{1 / 2}
\end{aligned}
$$




$$
\begin{array}{r}
\sigma_{\xi}=R_{m}(\tau)\left\{\frac{A^{2}}{(2 T m)^{2}} \int_{0}^{2 T m} d t^{\prime \prime} \int_{0}^{2 T m} d t^{\prime} \overline{n\left(t^{\prime}\right) n\left(t^{\prime \prime}\right)} \times\right. \\
\left.\sin w_{i}\left(t^{\prime}+\tau\right) \sin w_{i}\left(t^{\prime \prime}+\tau\right)\right\}^{1 / 2}
\end{array}
$$

For a control system designer, the power spectrum of the noise, rather than its correlation function, is more readily available. The correlation function of noise, $\overline{n\left(t^{\prime}\right) n\left(t^{\prime \prime}\right)}$, is related to the power spectrum of the noise, $\Phi_{n n}(w)$, by

$$
\overline{n\left(t^{\prime}\right) n\left(t^{\prime \prime}\right)}=\int_{0}^{\infty} \Phi_{n n}(w) \cos w\left(t^{\prime}-t^{\prime \prime}\right) d w \ldots
$$

By substitution of Fquation [34] into Equations [32] and [33], and carrying out the integrations with respect to $t^{\prime}$ and $t^{\prime \prime}$ the mean deviations $\sigma_{\zeta}$ and $\sigma_{\xi}$ now become

$$
\begin{aligned}
\sigma_{\xi}= & {\left[\frac{1}{T} \int_{0}^{2 T} R_{m}(\tau) \sin w_{i} \tau d \tau\right] \times } \\
& \left\{\frac{A^{2}}{\pi^{2} m^{2}} \int_{0}^{\infty} \Phi_{n n}(w) \frac{\left(w / w_{i}\right)^{2} \sin ^{2}\left(\pi m w / w_{i}\right)}{\left[\left(w / w_{i}\right)^{2}-1\right]^{2}} d w\right\}^{1 / 2} \cdots
\end{aligned}
$$

and

$$
\begin{gathered}
\sigma_{\xi}=R_{m}(\tau)\left\{\frac{A^{2}}{\pi^{2} m^{2}} \int_{0}^{\infty} \Phi_{n n}(w) \frac{\sin ^{2}\left(\pi m w / w_{i}\right)}{\left[\left(w / w_{i}\right)^{2}-1\right]^{2}} d w+\right. \\
\left.\frac{A^{2}}{\pi^{2} m^{2}} \sin ^{2} w_{i} \tau \int_{0}^{\infty} \Phi_{n n}(w) \frac{\sin ^{2}\left(\pi m w / w_{i}\right)}{\left(w / w_{i}\right)^{2}-1} d w\right\}^{1 / 2} \ldots
\end{gathered}
$$

When the first probability distributions of the statistical variables $\zeta$ and $\xi$ are not known, it is possible to make a broad approximation of the errors $\epsilon_{\zeta}$ and $\epsilon_{\xi}$, the probability of finding $\beta \beta^{\prime}<0$, and the probability of finding $l l^{\prime}<0$, respectively. First, for this purpose the probability of large deviation from the mean will be estimated.

Assume, for example, that the $\zeta$ 's distribute themselves around the mean $\bar{\zeta}$ symmetrically and that $\bar{\zeta}$ is positive and sufficiently large. Then the probability of the occurrence of large deviations from the mean is given by BienayméChebyshev's inequality (Ref. 3)

$$
P\left[|\zeta-\vec{\zeta}| \geq K \sigma_{\zeta}\right] \leq\left(1 / K^{2}\right) .
$$

The error $\epsilon_{\zeta}$ is one half of the probability given by Equation [37] when $K \sigma_{\zeta}$ is replaced by $\vec{\zeta}$. Thus

$$
\epsilon_{\zeta}=\frac{1}{2} P[|\zeta-\bar{\zeta}| \geq \bar{\zeta}] \leq \frac{1}{2}\left(\sigma_{\zeta} / \bar{\zeta}\right)^{2} \ldots
$$

Similarly, the error $\epsilon \xi$, the probability of finding $l l^{\prime}<0$, can be approximated as

$$
\epsilon_{\xi} \leq \frac{1}{2}\left(\sigma_{\xi} / \bar{\xi}\right)^{2}
$$

A sharper approximation of the errors $\epsilon_{\zeta}$ and $\epsilon_{\xi}$ will be obtained when the probability distribution is assumed to be unimodal. Then the Gauss inequality (3) can be used in place of the Bienaymé-Chebyshev inequality. In this case, the errors $\epsilon_{\xi}$ and $\epsilon_{\xi}$ are found to be

$$
\epsilon_{\zeta} \leq \frac{2}{9}\left(\sigma_{\zeta} / \bar{\zeta}\right)^{2} \ldots \ldots \ldots \ldots \ldots \ldots
$$

and

$$
\epsilon \xi \leq \frac{2}{9}\left(\sigma_{\xi} / \bar{\xi}\right)^{2}
$$

When the first probability distributions of $\zeta$ and $\xi$ are known, it is possible to compute $\epsilon_{\zeta}$ and $\epsilon_{\xi}$ exactly. For example, since $\zeta$ is a stationary random variable, its first probability distribution $W_{1}$ is a function of $\zeta$ only. Then the probability that $\zeta<0$ is simply the error $\epsilon_{\zeta}$; thus

$$
\epsilon_{\zeta}=\int_{-\infty}^{0} W_{1}(\zeta) d \zeta
$$

By the assumption of the first probability distribution of $\zeta$ as Gaussian, the error $\epsilon_{\zeta}$ is found to be

$$
\epsilon_{\zeta}=1 / 2\left[1-\operatorname{erf}\left(\frac{1}{\sqrt{2}} \frac{\bar{\zeta}}{\sigma_{\zeta}}\right)\right] \ldots
$$

Similarly, the error $\epsilon \xi$ is obtained as

$$
\epsilon \xi=1 / 2\left[1-\operatorname{erf}\left(\frac{1}{\sqrt{2}} \frac{\bar{\xi}}{\sigma_{\xi}}\right)\right]
$$

It is to be noted that whether the probability of the occurrence of Type II error is approximated, for example, by one of Equations [39] or [41], or is computed by assuming a first probability distribution, Equation [43], $\epsilon \xi$ depends only on the quotient $\bar{\xi} / \sigma_{\xi}$. Fig. 6 shows the probability of the occurrence of Type II error as a function of the quotient $\bar{\xi} / \sigma_{\xi}$ or $\bar{\zeta} / \sigma_{\zeta}$.

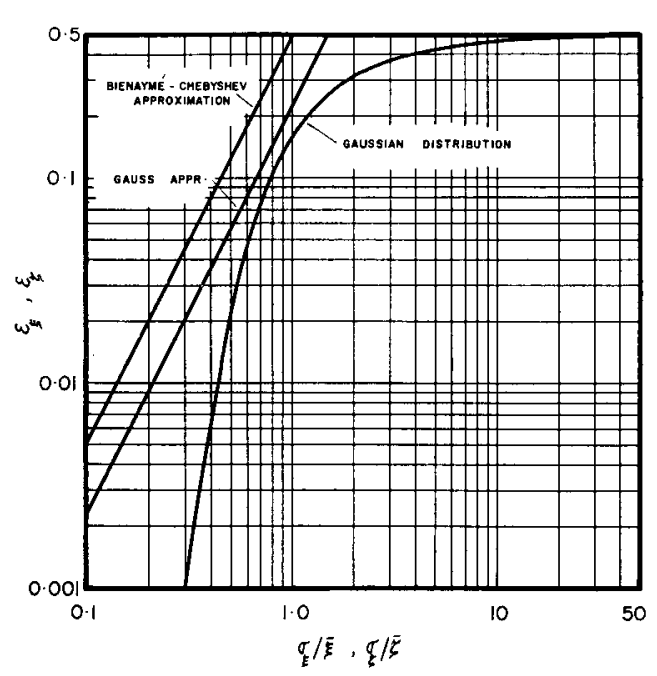

Fig. 6 Probability of occurrence of Type II error vs. the quotient $\sigma \xi / \bar{\xi}$ or $\sigma_{\zeta} / \bar{\zeta}$

In order to demonstrate a possible way of choosing the limiting parameter $l^{\prime *}$ or $\beta^{\prime *}$ when the probability of Type II error is assigned and the statistical properties of the noise interference are given, one can form the following quotients

$$
\begin{aligned}
& \beta^{\prime} \frac{\sigma_{\zeta}}{\bar{\zeta}}=\sqrt{\overline{n^{2}(t)}} A\left\{\frac{1}{\frac{1}{\pi^{2} m^{2}}} \frac{1}{\overline{n^{2}(t)}} \int_{0}^{\infty} \Phi_{n n}(w) \times\right. \\
& \left.\frac{\left(w / w_{i}\right)^{2} \sin ^{2}\left(\pi m w / w_{i}\right)}{\left[\left(w / w_{i}\right)^{2}-1\right]^{2}} d w\right\}^{1 / 2} \\
& l^{\prime} \frac{\sigma \xi}{\bar{\xi}}=\frac{\sqrt{n^{2}(t)}}{D} \frac{\pi^{2}}{2} \frac{1}{n^{2}(t)}\left\{\frac{1}{\pi^{2} m^{2}} \int_{0}^{\infty} \frac{\Phi_{n a}(w) \sin ^{2}\left(\pi m w / w_{i}\right)}{\left[\left(w / w_{i}\right)^{2}-1\right]^{2}} d w+\right. \\
& \left.\frac{\sin ^{2} w_{i} \tau}{\pi^{2} m^{2}} \int_{0}^{\infty} \frac{\Phi_{n n}(w) \sin ^{2}\left(\pi m w / w_{i}\right)}{\left(w / w_{i}\right)^{2}-1} d w\right\}^{1 / 2} \times \\
& {\left[\frac{1}{12}-\left(\tau_{i} / T\right)^{2}+2\left(\tau_{i} / T\right)^{3} \tanh \left(T / 2 \tau_{i}\right)\right]\left[1+\left(w_{i} \tau_{0}\right)^{2}\right]\left[1+\left(w_{i} \tau_{i}\right)^{2}\right]} \\
& \left\{\left(w_{i}^{2} \tau_{0} \tau_{i}-1\right) \cos w_{i} \tau+w_{i}\left(\tau_{0}+\tau_{i}\right) \sin w_{i} \tau\right\}
\end{aligned}
$$

where Equation [44] is obtained from Equations [30] and [35], and Equation [45] is obtained from Equations [21], [31], and [36]. The parameter $D$ is the hunting loss of the control system in the correct mode of operation.

When all system characteristics are available and when the power spectrum of the noise can be estimated, then $l^{\prime *}$ is obtained from Equation [45] by using the quotient $\sigma_{\xi} / \bar{\xi}$, which is obtained from Fig. 6 with the assigned value of $\epsilon \xi$. To illustrate a typical procedure, choose $\tau=0$; then Equation [45] 
becomes

$$
l^{\prime *}=\frac{\sqrt{\overline{n^{2}(t)}}}{D} \cdot \frac{\bar{\xi}}{\sigma_{\xi}} \cdot g \cdot f
$$

where

$$
g=\left\{\frac{1}{\pi^{2} m^{2}} \frac{1}{\overline{n^{2}(t)}} \int_{0}^{\infty} \Phi_{n n}(w) \frac{\sin ^{2}\left(\pi m w / w_{i}\right)}{\left[\left(w / w_{i}\right)^{2}-1\right]^{2}} d w\right\}^{1 / 2}
$$

and

$f=\frac{\pi^{2}}{2} \times$

$\left[\frac{1}{12}-\left(\tau_{i} / T\right)^{2}+2\left(\tau_{i} / T\right)^{3} \tanh \left(T / 2 \tau_{i}\right)\right]\left[1+\left(w_{i} \tau_{i}\right)^{2}\right]\left[1+\left(w_{i} \tau_{0}\right)^{2}\right]$

$$
\left(w_{i}^{2} \tau_{0} \tau_{i}-1\right)
$$

Fig. 7 shows the characteristic noise function $g$ as a function of the ratio $w_{n} / w_{i}$ with $m$ as parameter for typical noise power spectrum $\Phi_{n n}(w)=\gamma^{2} /\left(w_{n}^{2}+w^{2}\right)$; where $\gamma$ is a constant and $w_{n}$ is a characteristic noise frequency. From this figure it is observed that for $w_{n} / w_{i} \approx 1$, the function $g$ decreases in magnitude very rapidly for the first few values of the parameter $m$ and for the values of the quotient $w_{n} / w_{i}$ away from unity, the

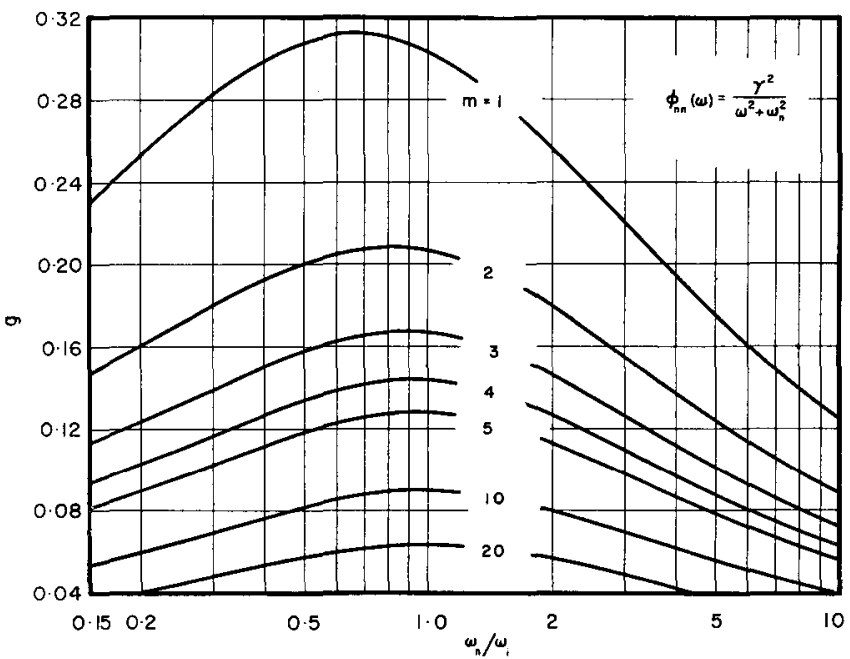

Fig. 7 Characteristic noise function $g$ vs. $w_{n} / w_{i}$ with $m$ as parameter for $\Phi_{n n}(w)=\gamma^{2} /\left(w_{n}^{2}+w^{2}\right)$

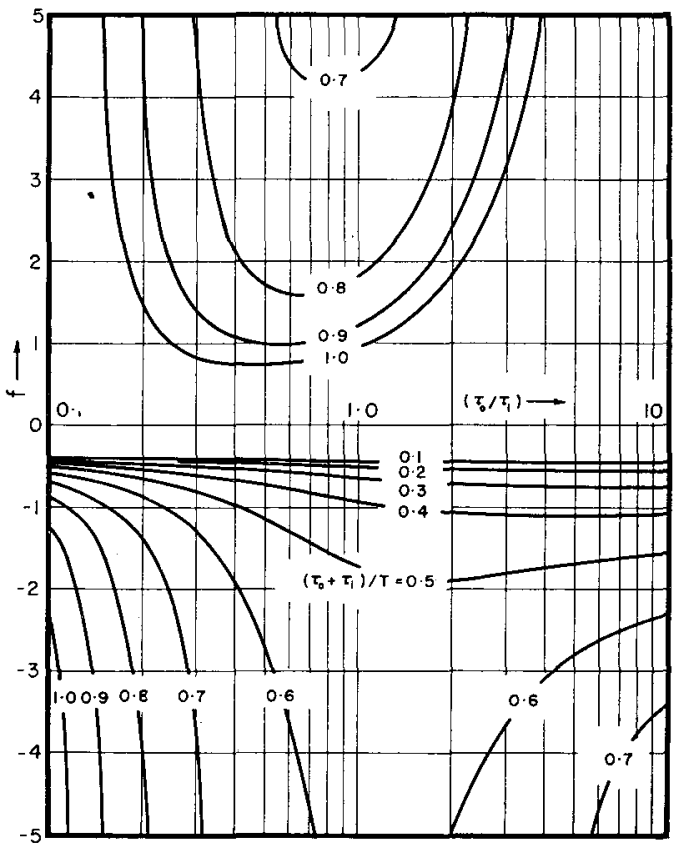

Fig. 8 Controlled system dynamic characteristics function $f$ vs. $\tau_{0} / \tau_{i}$ with $\left(\tau_{0}+\tau_{i}\right) / T$ as parameter effect of the parameter $m$ on the function $g$ is unimportant. Fig. 8 shows the controlled system dynamic characteristics function $f$ as a function of $\tau_{0} / \tau_{i}$ with $\left(\tau_{0}+\tau_{i}\right) / T$ as parameter. Figs. 9 and 10 show the limiting value $l^{\prime *}$ as a function of the dimensionless noise level $\sqrt{\overline{n^{2}(t)}} / D$ with $m$ as a parameter. On each of these figures, two values of $l^{\prime *}$ are presented which correspond to five and ten per cent probability of the occurrence of Type II error due to noise interference effects, which are characterized by a power spectrum of the form $\Phi_{n n}(w)=$ $\gamma^{2} /\left(w^{2}+w_{n}^{2}\right)$ and characteristic frequency $w_{n}=w_{i}$. Thus Figs. 6 through 10 are typical illustrations of the analysis which must be carried out to determine the parameter $l^{\prime *}$.

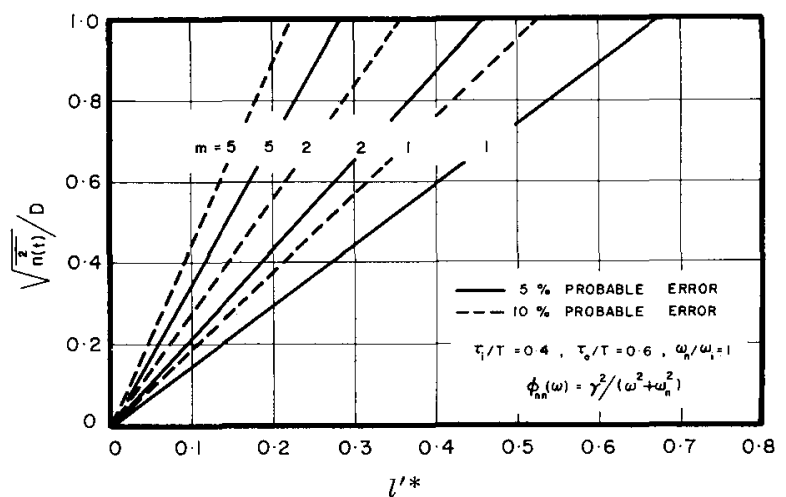

Fig. 9 Dimensionless noise level $\sqrt{\overline{n^{2}(t)}} / D$ vs. $l^{* *}$ with $m$ as parameter for $\tau_{i} / T=0.40, \tau_{0} / T=0.60, w_{n} / w_{i}=1$, and $\Phi_{n n}(w)=\gamma^{2} /\left(w n^{2}+w^{2}\right)$

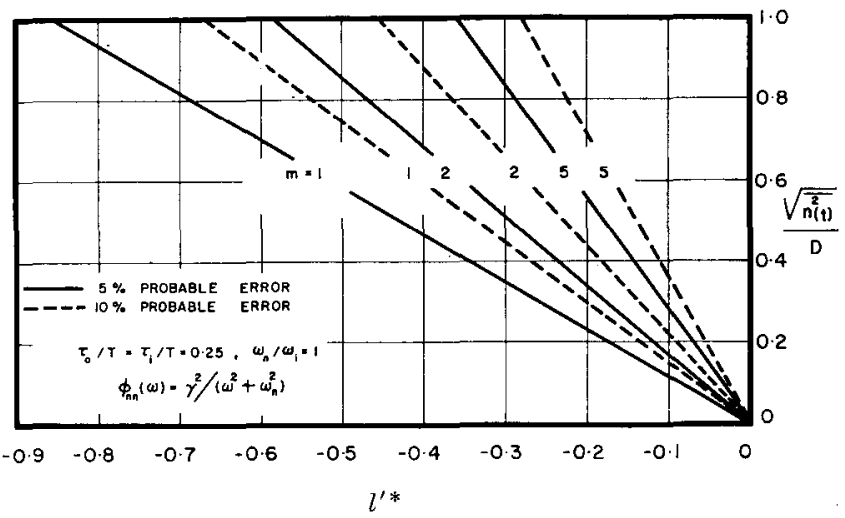

Fig. 10 Dimensionless noise level $\sqrt{\overline{n^{2}(t)}} / D$ vs. $l^{\prime *}$ with $m$ as parameter for $\tau_{i} / T=\tau_{0} / T=0.25, w_{n} / w_{i}=1$, and $\Phi_{n n}(w)=$ $\gamma^{2} /\left(w n^{2}+w^{2}\right)$

\section{Concluding Remarks}

In (2) it was indicated that the sawtooth form of input is the simplest type that is suitable for a peak-holding optimalizing control. However, in this work, the optimalizing control scheme is changed in such a manner that any periodic signal can be used as the input and the resulting control system will again hunt around the optimum state. As an illustration, one can consider a simple s.nusoidal form of "potential" input with an amount of incorrect input $b$, i.e.

$$
x_{\mathrm{s}}{ }^{*}=b+a^{\prime} \sin w_{i} t .
$$

If it is assumed that the input and output linear groups can be closely approximated by first order systems, then the actual input $x_{s}$, the actual output $y_{s}$, and the hunting loss $D_{s}$ in the incorrect mode of operation are found to be

$$
x_{s}=b+\frac{a^{\prime}}{1+\left(w_{i} \tau_{i}\right)^{2}}\left\{\sin w_{i} t-\left(w_{i} \tau_{i}\right) \cos w_{i} t\right\}
$$


$y_{s}=-k b^{2}-2 a^{\prime} b k \frac{\left[1-w_{i}^{2} \tau_{0} \tau_{i}\right] \sin w_{i} t-w_{i}\left(\tau_{0}+\tau_{i}\right) \cos w_{i} l}{\left[1+\left(w_{i} \tau_{0}\right)^{2}\right]\left[1+\left(w_{i} \tau_{i}\right)^{2}\right]}+$

$\frac{a^{\prime 2} k}{2}\left\{\frac{2\left[w_{i} \tau_{0}\left(1-w_{i}{ }^{2} \tau_{i}{ }^{2}\right)+w_{i} \tau_{i}\right] \sin 2 w_{i} t}{\left[1+\left(2 w_{i} \tau_{0}\right)^{2}\right]\left[1+\left(w_{i} \tau_{i}\right)^{2}\right]^{2}}-\frac{1}{1+\left(w_{i} \tau_{i}\right)^{2}}+\right.$

$$
\left.\frac{\left[1-\left(w_{i} \tau_{i}\right)^{2}-4 w_{i}{ }^{2} \tau_{i} \tau_{0}\right] \cos 2 w_{i t}}{\left[1+\left(2 w_{i} \tau_{0}\right)^{2}\right]\left[1+\left(w_{i} \tau_{i}\right)^{2}\right]^{2}}\right\}
$$

and

$$
D_{s}=k b^{2}+\frac{a^{\prime 2} k}{2} \frac{1}{1+\left(w_{i} \tau_{i}\right)^{2}}
$$

Similarly, the noise-interference-free cross-correlation function is obtained as

$$
\begin{aligned}
& \chi_{8}(\tau)=\frac{A a^{\prime} b k}{\left[1+\left(w_{i} \tau_{0}\right)^{2}\right]\left[1+\left(w_{i} \tau_{i}\right)^{2}\right]} \times \\
& \left\{\left(w_{i}^{2} \tau_{0} \tau_{i}-1\right) \cos w_{i} \tau+w_{i}\left(\tau_{0}+\tau_{i}\right) \sin w_{i} \tau\right\} \ldots \ldots[5
\end{aligned}
$$

It is observed that the form of Equation [51] is exactly the same as that of Equation [13]. The only difference is a multiplicative constant. Therefore it is unnecessary to carry out the remainder of the analysis for this type of control system. The fundamental concepts introduced in previous sections are perfectly valid in the present analysis.

The analyses which have been carried out so far have considered, for the sake of simplicity, a single output due to a single input. For some controlled systems to which the optimalizing control scheme can be applied, it may be necessary to consider several simultaneous inputs. In this case one has to consider a negative definite input-output characteristics surface in $n+1$ dimensions. For example, referred to the optimum point an operation surface of this sort may be defined as

$$
y^{*}=-\sum_{k=1}^{n} A_{k} x_{k}{ }^{2}+\sum_{\substack{k=1 \\(k \neq j)}}^{n} \sum_{\substack{j=1 \\(k \neq j)}}^{n} C_{k i} x_{k}{ }^{*} y_{i}{ }^{*} \ldots \ldots \ldots
$$

where $A_{k}$ and $C_{k i}\left(C_{k j} \neq C_{j k}\right)$ are characteristic constants of the controlled system, and $n$ is the number of inputs such that for all real values of $x^{*}, y^{*} \leq 0$. Therefore $x^{*}=0$ corresponds to the optimum point. For the purpose of illustration of the detection and elimination of incorrect mode of operation in this case, consider a sinusoidal input optimalizing control without dynamic effects. Thus, in the incorrect mode of operation each input $x_{k}^{*}$ has the form

$$
x_{k}^{*}=b_{k}+a_{k} \sin w_{k} t
$$

where $b_{k}$ is the amount of incorrect input of the $k$ th input, and each input hunting frequency $w_{k}$ is distinct. By substitution of Equation [53] into Equation [52], the output $y_{u}{ }^{*}$ due to incorrect mode of operation is obtained as

$$
\begin{aligned}
& y_{u}{ }^{*}= \\
& -\sum_{k=1}^{n} A_{k}\left\{\left(b_{k}{ }^{2}+1 / 2 a_{k}^{2}\right)+2 b_{k} a_{k} \sin w_{k} t-1 / 2 a_{k}^{2} \cos 2 w_{k} t\right\} \\
& +\sum_{\substack{k=1 \\
(k \neq j)}}^{n} \sum_{\substack{j=1 \\
(k \neq j}}^{n} C_{k j}\left\{b_{k} b_{j}+b_{j} a_{k} \sin w_{k} t+\right. \\
& \left.b_{k} a_{j} \sin w_{j} t+1 / 2 a_{k} a_{j}\left[\cos \left(w_{k}-w_{\jmath}\right) t-\cos \left(w_{k}+w_{j}\right) t\right]\right\} .
\end{aligned}
$$

In the correct mode of operation, all the $b_{k}$ are zero; thus the output in the correct mode of operation is

$$
\begin{aligned}
y^{*}= & -\sum_{k=1}^{n} A_{k}\left\{1 /{ }_{2} a_{k}{ }^{2}-1 / 2 a_{k}{ }^{2} \cos 2 w_{k} t\right\}+ \\
& \sum_{\substack{k=1 \\
(k \neq j)}}^{n} \sum_{j=1}^{n} 1 /{ }_{2} C_{k j} a_{k} a_{j}\left[\cos \left(w_{k}-w_{j}\right) t-\cos \left(w_{k}+w_{i}\right) t\right] \ldots
\end{aligned}
$$

It is observed from Equations [54] and [55] that the output contains components with frequency $w_{k}$ only in the incorrect mode of operation. Thus the detection criterion of the incorrect mode may be based on the existence of the components with frequencies $w_{k}$, when these frequencies are properly selected.

Since the controlled system may not respond properly to high-frequency variation of the inputs, the range of input hunting frequencies must not be very large. In particular, one should assign low frequencies to the inputs where their corresponding input linear groups have large characteristic time constants. When $n$ is not large, it may be sufficient to choose the ratio of the highest to the lowest input hunting frequencies to be equal to 2 , thus one may choose $\omega_{k}$ as

$$
w_{k} / w_{1}=[(n-1)+k] / n \quad(k=1,2, \ldots, n)
$$

Then, these assorted frequencies will never be equal to the combination frequencies, for example, $2 w_{k}, w_{k}-w_{j}$, and $\omega_{k}+\omega_{i}$, which can exist in both correct and incorrect modes of operation.

The incorrect inputs, the $b_{k}$, can be determined by either the correlation technique or by measuring the amplitudes of the components with frequencies $w_{k}$. In general there are obtained $k$ linear equations in the $b_{k}$ 's from which the $b_{k}$ may be determined. For the case of the correlation technique, the output should be cross-correlated simultaneously with signals of the form $\alpha_{k} \sin w_{k}\left(t+\tau_{k}\right)$. By taking the period of the cross-correlation process as $(2 n \pi) / w_{k}$, the contribution to the cross-correlation function will come from the particular component with frequency $w_{k}$. It is clear that the largest period of the cross-correlation is inversely proportional to the lowest input hunting frequency and this period increases as the number of inputs is increased.

\section{References}

1 Draper, C. S., and Li, Y. T., "Principles of Optimalizing Control Systems and an Application to the Internal Combustion Engine," The American Society of Mechanical Engineers Publicam tion, Sept. 1955.

2 Tsien, H. S., and Serdengecti, S., "Analysis of Peak-Holding Optimalizing Control," Journal of the Aeronautical Sciences, vol. 22, August 1955 , pp. $561-570$.

3 Cramér, H., "Mathematical Methods of Statistics," Princeton University Press, 1951, p. 183. 